\title{
Review Article \\ Breastfeeding Practices among Native Hawaiians and Pacific Islanders
}

\author{
Ingrid K. Richards Adams, ${ }^{1}$ Chizimuzo T. C. Okoli, ${ }^{2}$ Akilah Dulin Keita, ${ }^{3}$ \\ Ana Maria Linares, ${ }^{2}$ Keiko Tanaka, ${ }^{4}$ Joshua R. Polanin, ${ }^{5}$ and Annie Koempel ${ }^{6}$ \\ ${ }^{1}$ Department of Dietetics and Human Nutrition, University of Kentucky, 118 Funkhouser Building, Lexington, KY 40506, USA \\ ${ }^{2}$ University of Kentucky, 315 CON Building, Lexington, KY 40536, USA \\ ${ }^{3}$ Institute for Community Health Promotion, Department of Behavioral and Social Sciences, Brown University, \\ Box G-S121-8, Providence, RI 02908, USA \\ ${ }^{4}$ University of Kentucky, Lexington, KY, USA \\ ${ }^{5}$ Peabody Research Institute, Vanderbilt University, Nashville, TN, USA \\ ${ }^{6}$ Department of Dietetics and Human Nutrition, University of Kentucky, Lexington, KY, USA
}

Correspondence should be addressed to Ingrid K. Richards Adams; ingrid.adams@uky.edu

Received 4 April 2016; Accepted 25 August 2016

Academic Editor: Li Ming Wen

Copyright (C) 2016 Ingrid K. Richards Adams et al. This is an open access article distributed under the Creative Commons Attribution License, which permits unrestricted use, distribution, and reproduction in any medium, provided the original work is properly cited.

\begin{abstract}
Background. Breastfeeding is associated with a decreased risk of obesity in the early and adult years. Native Hawaiians and Pacific Islanders (NHPI) experience high rates of obesity which is often obfuscated with aggregated data. Using disaggregated data, we examined breastfeeding practices among NHPI. Methods. Seven databases and reference lists were searched. Two independent researchers extracted relevant studies based on predetermined criteria. Nine studies met our inclusion criteria and a meta-analysis was conducted using random-effects, inverse-various weighted models. Results. Few studies disaggregated NHPI populations when examining breastfeeding practices. Most studies were cross-sectional and our search yielded no randomized or quasirandomized control trials. The results of the meta-analysis indicated that $46.5 \%$ NHPI women initiated breastfeeding with $40.8 \%$ breastfeeding exclusively. These pooled analyses show that NHPI breastfeeding practices are below the recommended national and international goals and guidelines. Conclusion. Breastfeeding practices among NHPI are heterogeneous and critical disparities exist among certain NHPI subgroups and additional research needs to be conducted to determine the reasons for the disparity. Future studies should work to disaggregate data for NHPI and the various subpopulations. Multicomponent, multilevel strategies are needed to support breastfeeding practices among NHPI.
\end{abstract}

\section{Introduction}

Obesity prevention begins with breastfeeding [1] and infancy (0 to 3 years) is a critical period in obesity development [2]. Recent research suggests a $15 \%$ to $30 \%$ reduction in adolescent and adult obesity rates with breastfeeding during infancy compared with none [1,3-7]. Breastfeeding's effects are dose dependent, with exclusive breastfeeding (EBF) and breastfeeding for long durations offering increased benefits [4, 8-13], including several short- and long-term medical, neurodevelopmental, and immunological cognitive advantages [14-19]. Due to these benefits, EBF is recommended for a minimum of six months after birth, followed by continued breastfeeding for a minimum of one year [1, 20-23].

Native Hawaiian and Pacific Islanders (NHPI) are a rapidly growing population and constitute approximately 1.2 million of the Asian American population either alone (44\%) or in combination with other races (56\%) [24]. Because NHPI are often conflated with Asian Americans and other Pacific Islanders in national and state-level data, disparities among them are often unnoticed. For example, when breastfeeding data are disaggregated by race and ethnicity, studies show that NHPI have lower breastfeeding initiation and EBF rates and have shorter breastfeeding duration than 
other populations [25-27]. This is concerning because NHPI populations experience higher rates of obesity and obesityrelated comorbidities and mortality than other populations, but improving breastfeeding practices can ameliorate obesityrelated issues [28-34]. Although steps have been taken to separate Asian and NHPI in census data, the problem of aggregating these groups is still prevalent [35].

Currently, there are no systematic reviews on breastfeeding practices that disaggregate data on NHPI. The information from such a systematic review can provide policy makers, researchers, and public health workers a means to support and enhance breastfeeding practices among NHPI while addressing the problem of high obesity. Therefore, the objectives of this study are to provide a systematic review and meta-analysis of breastfeeding practices used by NHPI in terms of initiation, EBF, and duration.

\section{Methods}

Searches were conducted in July 2013, October 2014, and updated in January 2016 in the online bibliographic databases PubMed, AGRICOLA, CENTRAL, CINAHL, PsychInfo, Sociological Abstracts, and Web of Knowledge for studies examining breastfeeding practices among NHPI. We used Medical Subject Heading (MeSH) terms in PubMed and other indexing terms for the respective databases, as well as text wording. Our initial searches combined the following terms: breast feeding, breast-feeding, breastfeeding, breastfed, breast fed, breast-fed, child, preschool, infant, Pacific Island, Oceanic ancestry group, Hawaii, breastfeed, bottle feeding, formula fed, formula milk, human milk, infant feeding, weaning, child preschool, infant, and pregnancy. We did not include language or date restrictions in our searches. Two researchers ( $I A$ and $A K$ ) independently scanned the reference lists of all relevant papers retrieved and extracted relevant studies using predetermined inclusion and exclusion criteria. Discrepancies were resolved through discussion so that consensus could be attained.

2.1. Inclusion. Studies were included if data were disaggregated for NHPI populations and if they were directly related to breastfeeding practices. Excluded articles were those that were conducted outside of the United States or the Pacific Islands (Micronesia, Melanesia, and Polynesia) or qualitative in nature.

2.2. Coding. Two researchers independently coded studies for type, sample size, outcome measures, and effect size. The effect size of interest was the proportion of mothers who adopted certain breastfeeding practices (initiation, exclusive breastfeeding, and duration) or children on whom these practices were used.

2.3. Synthesis. We performed two synthesis techniques: (1) a narrative report of breastfeeding practices among NHPI samples in all studies and (2) a meta-analysis of various breastfeeding practice measures using a random-effects, inversevarious weighted model, with effect sizes grouped according to similar measurement constructs. For example, we synthesized measures of breastfeeding initiation separately from breastfeeding duration and exclusivity. Heterogeneity among the effect sizes was calculated using $I^{2}$ index [43]. All analyses were conducted using R package metafor [44].

\section{Systematic Review}

We identified 718 articles from which we removed 336 duplicates; and, of 382 articles screened for potential relevance, 348 were excluded. Thirty-four articles were assessed for eligibility. Further studies were excluded because they (1) were outside of the United States or Pacific Islands $(N=8)$, (2) addressed complementary feeding or did not disaggregate NHPI from other ethnic groups $(N=16)$, (3) described interventions (without reporting effects) $(N=1)$, and were secondary data from other included studies. Hence, nine studies were included in our final results. See Figure 1.

\section{Main Findings of the Selected Studies}

Study dates ranged from 1969 to 2013. Six studies were conducted before the year 2000. Studies were cross-sectional $[26,27,36,37,39,41]$ and each one was cohort [38], panel [40], and univariate descriptive [42]. Furthermore, just over half of the studies were conducted in Hawaii or used Hawaiian datasets $[26,27,36,39,41]$, and six focused on ethnically diverse populations [26, 27, 36, 39-41], four only on populations in the Pacific Islands [37, 38, 40, 42], and one solely on Native Hawaiians [27]. See Table 1.

\section{Breastfeeding Initiation}

Four studies examined breastfeeding initiation, three among Hawaiians and one in the Pacific Islands. In the first study, an initiation rate of $29.0 \%$ for Hawaiian mothers was observed as compared to $26.0 \%$ Caucasians and $50.0 \%$ Japanese [36]. In the second study, a $52.6 \%$ breastfeeding rate at hospital discharge was observed for Native Hawaiian women as compared to $70.4 \%$ Caucasian, 55.4\% Japanese, and 33.3\% Filipino [39]. In the third study, a 1-month initiation rate of 91.0\% was observed for Native Hawaiian mothers attending WIC clinics [27]. Finally, among Pacific Islanders, all mothers on Honiara and Nggela-Sandfly breastfed their children [37]. The results of the meta-analysis indicated that less than half of mothers initiated breastfeeding ( $\mathrm{M}=46.5 \%$, 95\% CI (26.966.1), $I^{2}=95.22$ ).

\section{Exclusive Breastfeeding}

Six studies reported on EBF $[26,27,38,40-42]$ with three in Hawaii and three in the Pacific Islands. The first study among only Native Hawaiians, reported 3- and 6-month EBF rates of $29.0 \%$ and $18.0 \%$, respectively [27]. The second study showed a $31.8 \%$ EBF rate among Hawaiian mothers as compared to $51.9 \%$ White, $38.0 \%$ Korean, 37.0\% Black, 33.8\% Chinese, 29.4\% Japanese, 28.9.4\% Filipino, and 24.2\% Samoan [26]. The third study found an $18.4 \%$ EBF rate among Hawaiians 


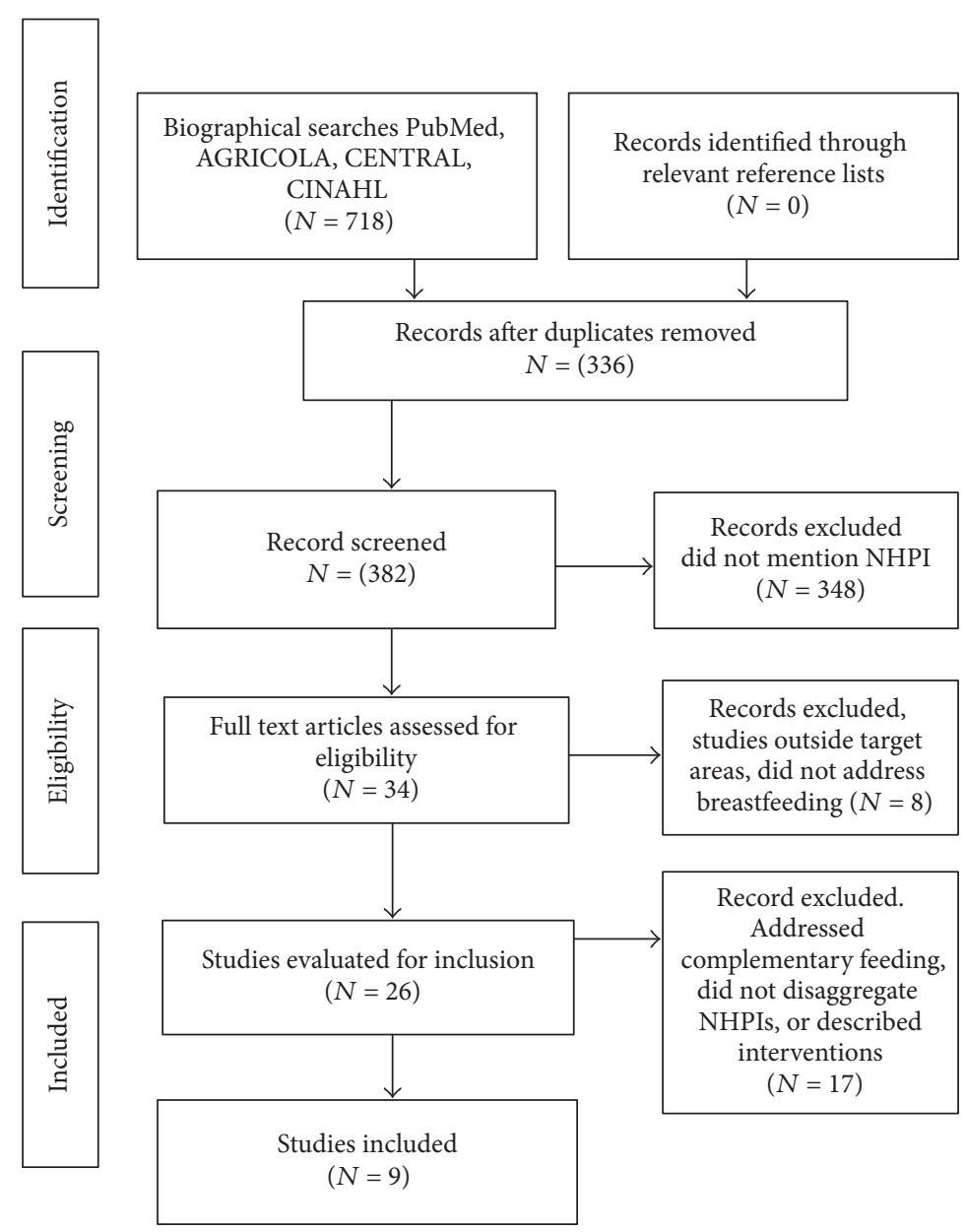

FIGURE 1: Prisma flow diagram of selected studies.

but did not disaggregate the data by ethnicity [41]. The fourth study in Fiji reported EBF rates of $68.5 \%$ at 3 months [40]. However, a decline was shown from $60.2 \%$ in 1977 to $45.6 \%$ in 1980. The fifth study in Kiribati reported EBF rates of $92.0 \%$ at 4 months [38]. The final study in the Commonwealth of the Northern Mariana Islands also found a decline in EBF rates with $46.0 \%, 23.0 \%, 26.0 \%$, and $9.0 \%$ of children exclusively breastfed for 0-2 months, 2-4 months, 4-6 months, and 9 or more months, respectively [42]. Meta-analysis results showed that less than half of mothers reported EBF practices $(\mathrm{M}=$ $40.8 \%$, 95\% CI (17.8-63.7), $\left.I^{2}=99.74\right)$.

\section{Breastfeeding Duration}

Four studies in our review measured breastfeeding duration, three among Pacific Islanders and one among Native Hawaiians. The first study observed that $45.7 \%$ of all infants in Kiribati were breastfed at 12 months [38]. The second study among mothers in the Commonwealth of the Northern Marianas Island reported that $22.0 \%$ received breastmilk for 12 or more months [42]. The third study [37] mentioned that most mothers in both Honiara and Nggela-Sandfly were breastfeeding at 6 and 12 months. The fourth study in Oahu [27] reported a 6-month breastfeeding duration rate of $52.0 \%$ for all mothers. Due to the variability in measures of breastfeeding duration a meta-analysis could not be appropriately performed.

\section{Discussion}

The high incidence of obesity, low breastfeeding rates, and other chronic diseases among NHPI makes it imperative to study differences in breastfeeding practices in these populations. Our review found that breastfeeding practices among NHPI are heterogeneous, highlighting disparities in breastfeeding practices among certain NHPI subgroups [26]. Examining breastfeeding practices among NHPI can expose hidden health issues and trends, making it possible to unearth disparity [45].

Our systematic review yielded only nine studies spanning a 44-year period. Moreover, we found limited disaggregated data in national surveys and surveillance systems for these groups. We found no studies examining breastfeeding patterns and disaggregating data for NHPI in the US where approximately two-thirds of NHPI reside [34]. The NHPI population is a young population that is expected to increase 


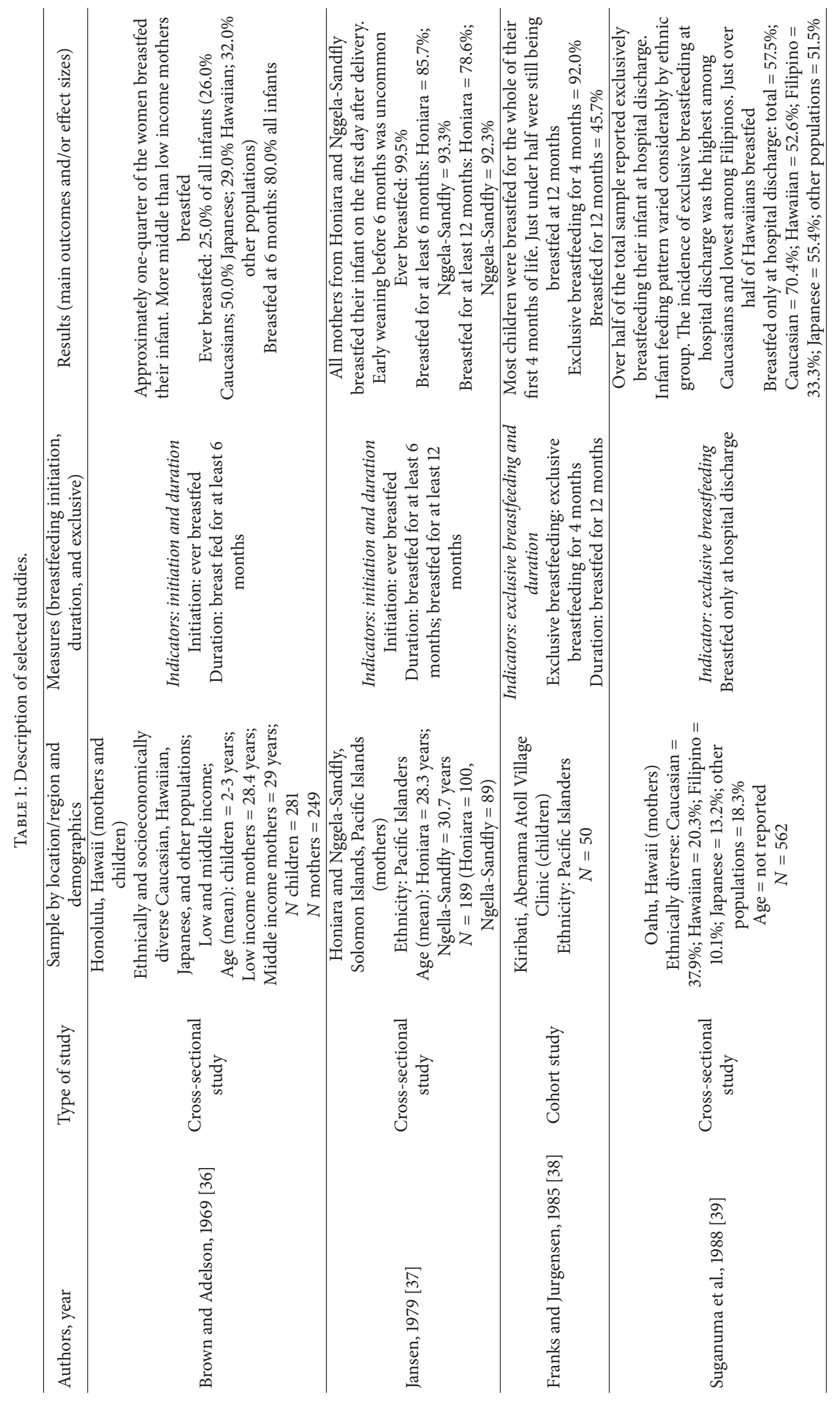




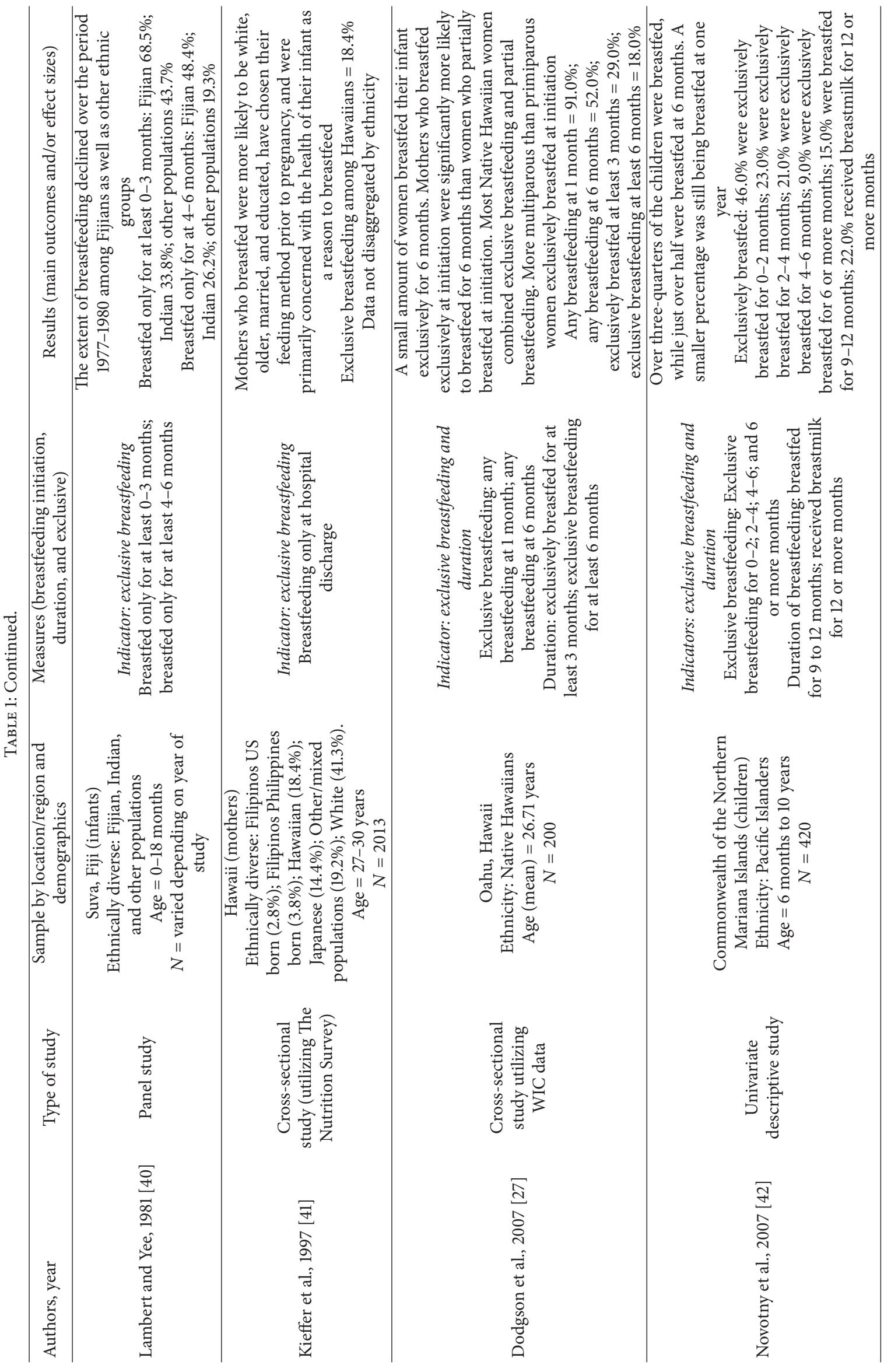




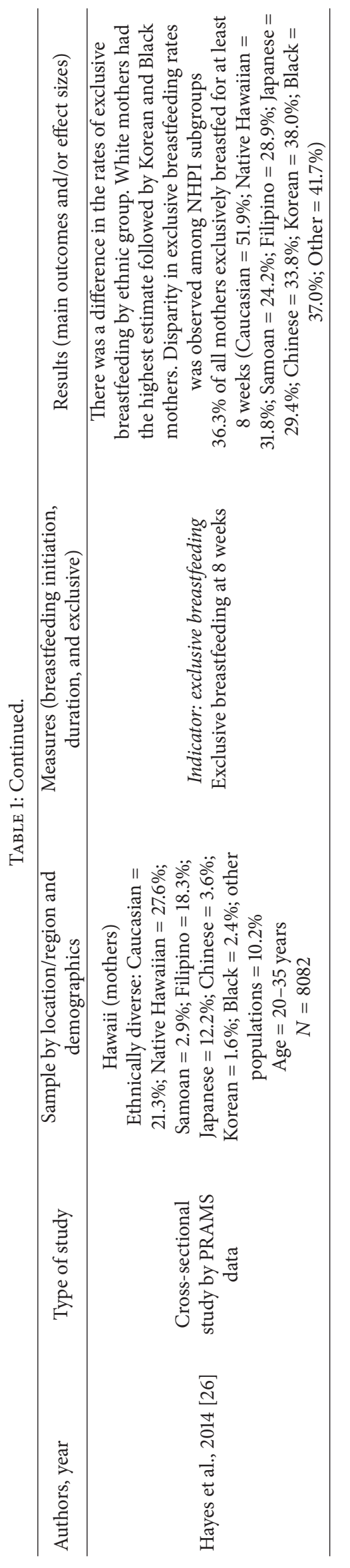


exponentially by the year $2060[46,47]$, and it is also critical that mandates related to disaggregating NHPI data by distinct ethnic and racial groups separate from Asian Americans are implemented $[34,46]$. There is a need to enhance the monitoring of breastfeeding practices in all US NHPI states, territories, and commonwealths to better identify risk factors for poor breastfeeding outcomes.

In addition, we found that varying terms and time periods were used for breastfeeding initiation, exclusivity, and duration among studies in our review. Such variations have also been observed among federally funded datasets, indicating a need for standardization of breastfeeding outcomes [45]. Future studies should implement consistent terminology for the purpose of comparing effects and replicating studies. In addition, it is important that studies use time frames (breastfeeding at 6 and 12 months and exclusive breastfeeding at 3 and 6 months) that are aligned with national objectives and national data collection and monitoring systems.

The result of our meta-analysis indicated that less than half of NHPI women initiated breastfeeding or breastfed exclusively. These pooled analyses show that NHPI breastfeeding practices are below the Healthy People 2020 goals. Qualitative studies have highlighted several factors that may affect breastfeeding practices among NHPI, namely, lack of knowledge, lactation problems, poor family and social support, social norms, embarrassment related to breastfeeding in public, employment and child care issues, and the education of health care providers, so that they can fully support the breastfeeding efforts of NHPI mothers [35, 48, 49]. Further research needs to be conducted to determine the reasons for the disparity in breastfeeding practices. We suggest, at a minimum, disaggregating survey data by the race/ethnicity in order that future analyst can use such data. Our search produced neither randomized nor quasirandomized controlled trials related to breastfeeding among NHPI. Studies are also needed to systematically explore the types of interventions that can enhance exclusive breastfeeding rates and duration among NHPI.

There are few important considerations that need to be noted in interpreting the findings of this review. The unclear definitions of breastfeeding terms, variability in the measurement periods, and the small number of studies are limitations. However, despite these concerns, the need for this study cannot be overemphasized as it illuminates disparities within the NHPI populations and brings to light the needs of a group that could potentially remain on the outskirts of policy decisions and focus.

\section{Conclusion}

Future studies should work to disaggregate NHPI data and identify barriers to breastfeeding among NHPI. Moreover, multicomponent, multilevel strategies directed toward NHPI communities are needed to support breastfeeding practices. Such studies can support existing culturally appropriate practices, advance health promotion activities, and provide directions for policies to minimize health-related morbidity, mortality, and disparities among the NHPI population.

\section{Competing Interests}

No competing financial interests exist.

\section{Acknowledgments}

This study was supported by the Robert Wood Johnson Foundation: New Connections Grant no. 19652. The authors would like to thank Dr. Rachel Novotny for her advice and suggestions during the preparation of the manuscript.

\section{References}

[1] American Academy of Pediatrics, "Breastfeeding and the use of human milk," Pediatrics, vol. 129, no. 3, pp. e827-e841, 2012.

[2] N. L. Hawley, W. Johnson, O. Nu'Usolia, and S. T. McGarvey, "The contribution of feeding mode to obesogenic growth trajectories in American Samoan infants," Pediatric Obesity, vol. 9, no. 1, pp. el-e13, 2014.

[3] K. E. Bergmann, R. L. Bergmann, R. Von Kries et al., "Early determinants of childhood overweight and adiposity in a birth cohort study: role of breast-feeding," International Journal of Obesity and Related Metabolic Disorders, vol. 27, no. 2, pp. 162172, 2003.

[4] T. Harder, R. Bergmann, G. Kallischnigg, and A. Plagemann, "Duration of breastfeeding and risk of overweight: a metaanalysis," American Journal of Epidemiology, vol. 162, no. 5, pp. 397-403, 2005.

[5] S. Ip, M. Chung, G. Raman, T. A. Trikalinos, and J. Lau, "A summary of the agency for healthcare research and quality's evidence report on breastfeeding in developed countries," Breastfeeding Medicine, vol. 4, supplement 1, pp. S17-S30, 2009.

[6] R. Kelishadi and S. Farajian, "The protective effects of breastfeeding on chronic non-communicable diseases in adulthood: a review of evidence," Advanced Biomedical Research, vol. 3, no. 1, article 3, 2014.

[7] M. W. Metzger and T. W. McDade, "Breastfeeding as obesity prevention in the United States: a sibling difference model," American Journal of Human Biology, vol. 22, no. 3, pp. 291-296, 2010.

[8] C. G. Owen, R. M. Martin, P. H. Whincup, G. D. Smith, and D. G. Cook, "Effect of infant feeding on the risk of obesity across the life course: a quantitative review of published evidence," Pediatrics, vol. 115, no. 5, pp. 1367-1377, 2005.

[9] A. D. Liese, T. Hirsch, E. Von Mutius, U. Keil, W. Leupold, and S. K. Weiland, "Inverse association of overweight and breast feeding in 9 to 10 -y-old children in Germany," International Journal of Obesity, vol. 25, no. 11, pp. 1644-1650, 2001.

[10] R. Von Kries, B. Koletzko, T. Sauerwald et al., "Breast feeding and obesity: cross sectional study," British Medical Journal, vol. 318, no. 7203, pp. 147-150, 1999.

[11] M. L. Hediger, M. D. Overpeck, R. J. Kuczmarski, and W. J. Ruan, "Association between infant breastfeeding and overweight in young children," The Journal of the American Medical Association, vol. 285, no. 19, pp. 2453-2460, 2001.

[12] J. Armstrong and J. J. Reilly, "Breastfeeding and lowering the risk of childhood obesity," The Lancet, vol. 359, no. 9322, pp. 20032004, 2002. 
[13] G. Neyzi, P. Binyildiz, and H. Gunox, "Influence of feeding pattern in early infancy on ponderal index and relative weight," in Human Growth and Development, K. Borms, Ed., pp. 603-611, Plenum, New York, NY, USA, 1984.

[14] "Breastfeeding and the use of human milk," Pediatrics, vol. 115, no. 2, article 496, 2012.

[15] A. Hörnell, H. Lagström, B. Lande, and I. Thorsdottir, "Breastfeeding, introduction of other foods and effects on health: a systematic literature review for the 5th Nordic Nutrition Recommendations," Food \& Nutrition Research, vol. 57, 2013.

[16] M. S. Kramer, F. Aboud, E. Mironova et al., "Breastfeeding and child cognitive development: new evidence from a large randomized trial," Archives of General Psychiatry, vol. 65, no. 5, pp. 578-584, 2008.

[17] M. A. Quigley, C. Hockley, C. Carson, Y. Kelly, M. J. Renfrew, and A. Sacker, "Breastfeeding is associated with improved child cognitive development: A Population-Based Cohort Study," Journal of Pediatrics, vol. 160, no. 1, pp. 25-32, 2012.

[18] M. B. Belfort, S. L. Rifas-Shiman, K. P. Kleinman et al., "Infant feeding and childhood cognition at ages 3 and 7 years: effects of breastfeeding duration and exclusivity," JAMA Pediatrics, vol. 167, no. 9, pp. 836-844, 2013.

[19] B. Frederiksen, M. Kroehl, M. Lamb et al., "Infant exposures and development of Type 1 Diabetes Mellitus: the diabetes autoimmunity study in the young (DAISY)," JAMA Pediatrics, vol. 167, no. 9, pp. 808-815, 2013.

[20] Breastfeeding, World Health Organization 2014, http://www .wpro.who.int/mediacentre/factsheets/nutrition_breastfeeding/ en/.

[21] Nutrition Exclusive Breastfeeding, World Health Organization, December 2012, http://www.who.int/nutrition/topics/ exclusive_breastfeeding/en/.

[22] United States Breastfeeding Committee, 2015, http://www .usbreastfeeding.org/.

[23] “Healthy People 2020," United States Department of Health and Human Services, https://www.healthypeople.gov/2020/ topics-objectives/topic/maternal-infant-and-child-health/ objectives.

[24] Department of Native Hawaiian Health, Center for Native and Pacific Health Disparities Research, John A. Burns School of Medicine, 2013.

[25] PRAMS Data on Breastfeeding, Center for Disease Control and Prevention, 2015, http://www.cdc.gov/prams/data-breastfeeding .htm.

[26] D. K. Hayes, K. M. Mitchell, C. Donohoe-Mather, R. L. Zaha, C. Melcher, and L. J. Fuddy, "Predictors of exclusive breastfeeding at least 8 weeks among asian and native Hawaiian or other Pacific Islander race subgroups in Hawaii, 2004-2008," Maternal and Child Health Journal, vol. 18, no. 5, pp. 1215-1223, 2014.

[27] J. E. Dodgson, E. Codier, P. Kaiwi, M. F. M. Oneha, and I. Pagano, "Breastfeeding patterns in a community of native Hawaiian mothers participating in WIC," Family and Community Health, vol. 30, no. 2, pp. S46-S58, 2007.

[28] L. D. McCubbin and M. Antonio, "Discrimination and obesity among native Hawaiians," Hawai'i Journal of Medicine \& Public Health, vol. 71, no. 12, pp. 346-352, 2012.
[29] "Hawaii Physical Activity and Nutrition Plan, 2013-2020," Hawaii State Department of Health, http://health.hawaii.gov/ physical-activity-nutrition/files/2013/08/Hawaii-PAN-Plan-20132020.pdf.

[30] Global Health Observatory Data Repository: Obesity Data by Country, World Health Organization (WHO), 2014, http://apps.who.int/gho/data/node.main.A900A?lang=en.

[31] C. L. Ogden, M. D. Carroll, B. K. Kit, and K. M. Flegal, "Prevalence of childhood and adult obesity in the United States, 2011-2012," The Journal of the American Medical Association, vol. 311, no. 8, pp. 806-814, 2014.

[32] G. Baruffi, C. J. Hardy, C. I. Waslien, S. J. Uyehara, and D. Krupitsky, "Ethnic differences in the prevalence of overweight among young children in Hawaii," Journal of the American Dietetic Association, vol. 104, no. 11, pp. 1701-1707, 2004.

[33] R. Novotny, C. E. S. Oshiro, and L. R. Wilkens, "Prevalence of childhood obesity among young multiethnic children from a health maintenance organization in Hawaii," Childhood Obesity, vol. 9, no. 1, pp. 35-42, 2013.

[34] Profile: Native Hawaiians and Pacific Islanders, U.S. Department of Health and Human Services, Office of Minority Health, 2015, http://minorityhealth.hhs.gov/omh/browse .aspx?lvl=3\&lvlid $=65$.

[35] Native Hawaiian and Pacific Islanders and a community of contrasts in the United States, Empowering Pacific Island Communities and Asian Americans Advancing Justice, 2014, http://empoweredpi.org/wp-content/uploads/2014/06/A_ Community_of_Contrasts_NHPI_US_2014-1.pdf.

[36] M. L. Brown and S. F. Adelson, "Infant feeding practices among low and middle income families in Honolulu," Tropical and Geographical Medicine, vol. 21, no. 1, pp. 53-61, 1969.

[37] A. Jansen, "Malnutrition and child-feeding practices among Solomon Islanders in Honiara and Nggela-Sandfly," The Journal of Tropical Pediatrics and Environmental Child Health, vol. 25, no. 1, pp. 15-22, 1979.

[38] A. J. Franks and C. Jurgensen, "Nutrition and health in the first year of life on a Pacific atoll. Observations on Abemama Atoll, Central Pacific," Transactions of the Royal Society of Tropical Medicine and Hygiene, vol. 79, no. 5, pp. 681-684, 1985.

[39] E. K. Suganuma, G. R. Alexander, G. Baruffi, and S. R. Gilden, "Infant feeding practices in Hawaii," Hawaii Medical Journal, vol. 47, no. 3, pp. 112-119, 1988.

[40] J. Lambert and V. Yee, "Suva infant feeding survey," Fiji Medical Journal, vol. 9, no. 1-2, pp. 5-9, 1981.

[41] E. C. Kieffer, R. Novotny, K. B. Welch, J. M. Mor, and M. Thiele, "Health practitioners should consider parity when counseling mothers on decisions about infant feeding methods," Journal of the American Dietetic Association, vol. 97, no. 11, pp. 1313-1316, 1997.

[42] R. Novotny, P. Coleman, L. Tenorio et al., "Breastfeeding is associated with lower body mass index among children of the commonwealth of the Northern Mariana Islands," Journal of the American Dietetic Association, vol. 107, no. 10, pp. 1743-1746, 2007.

[43] J. P. T. Higgins and S. G. Thompson, "Quantifying heterogeneity in a meta-analysis," Statistics in Medicine, vol. 21, no. 11, pp.15391558, 2002. 
[44] W. Viechtbauer, "Conducting meta-analyses in $\mathrm{R}$ with the metafor," Journal of Statistical Software, vol. 36, no. 3, pp. 1-48, 2010.

[45] D. J. Chapman and R. Pérez-Escamilla, "US national breastfeeding monitoring and surveillance: current status and recommendations," Journal of Human Lactation, vol. 25, no. 2, pp. 139-150, 2009.

[46] Asian Americans Advancing Justice, 2015, http://www .advancingjustice.org/.

[47] L. Hixson, B. Hepler, and K. Myoung, The Native Hawaiian and Other Pacific Islander Population: 2010, 2012, http://www .census.gov/prod/cen2010/briefs/c2010br-12.pdf.

[48] Centers for Disease Control and Prevention, Strategies to Prevent Obesity and Other Chronic Diseases: The CDC Guide to Strategies to Support Breastfeeding Mothers and Babies, U.S. Department of Health and Human Services, Atlanta, Ga, USA, 2013.

[49] M. Oneha and J. Dodgson, "Community influences on breastfeeding described by Native Hawaiian mothers," Pimatisiwin, vol. 7, no. 1, pp. 75-97, 2009. 


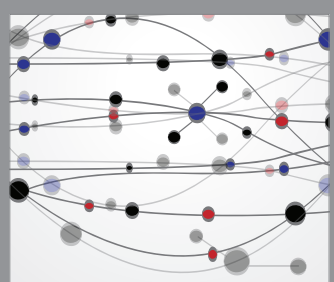

The Scientific World Journal
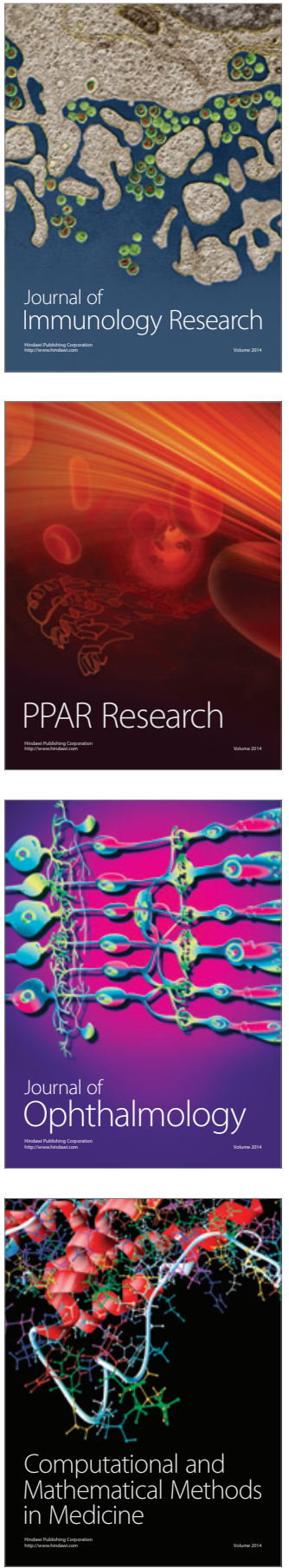

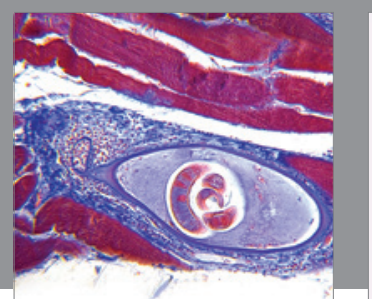

Gastroenterology Research and Practice

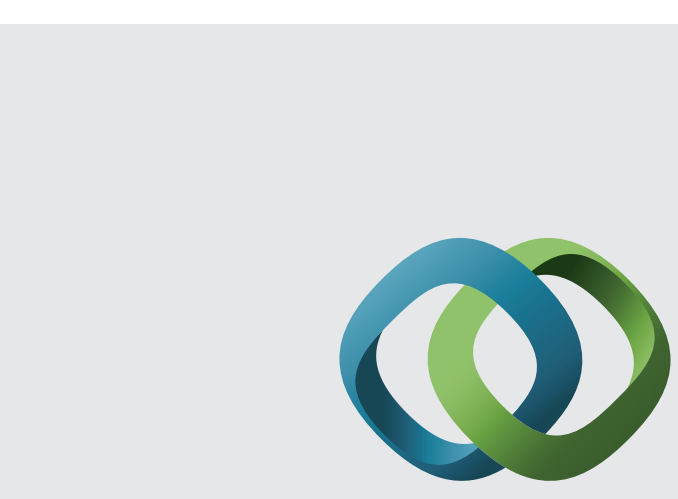

\section{Hindawi}

Submit your manuscripts at

http://www.hindawi.com
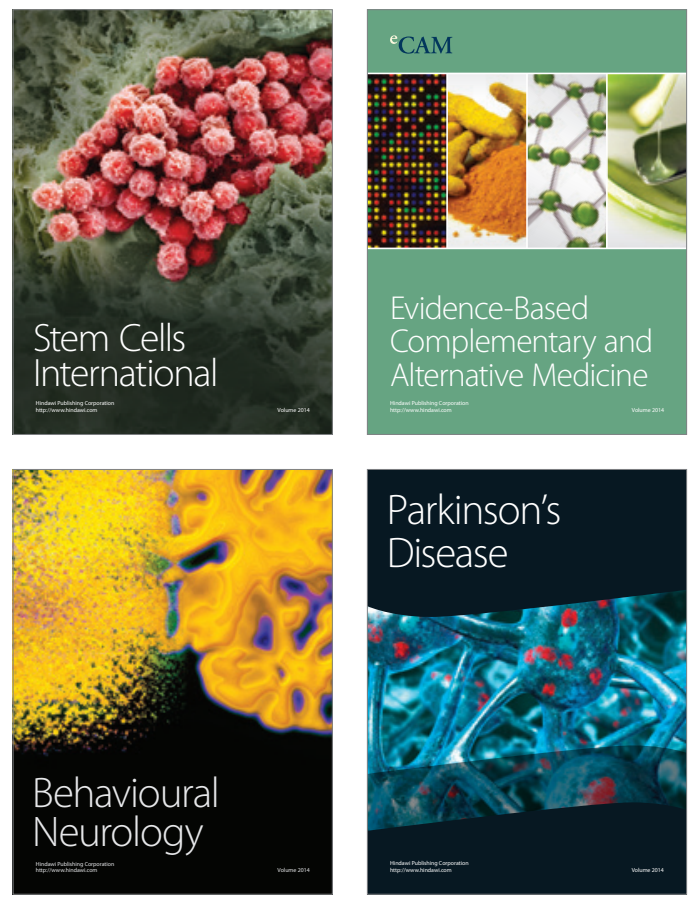
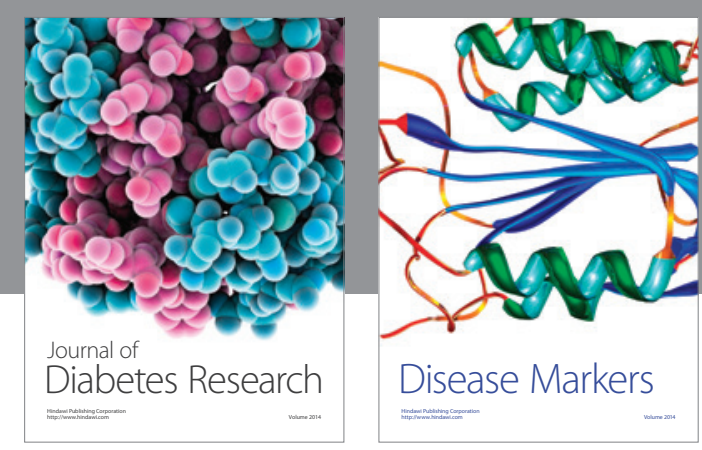

Disease Markers
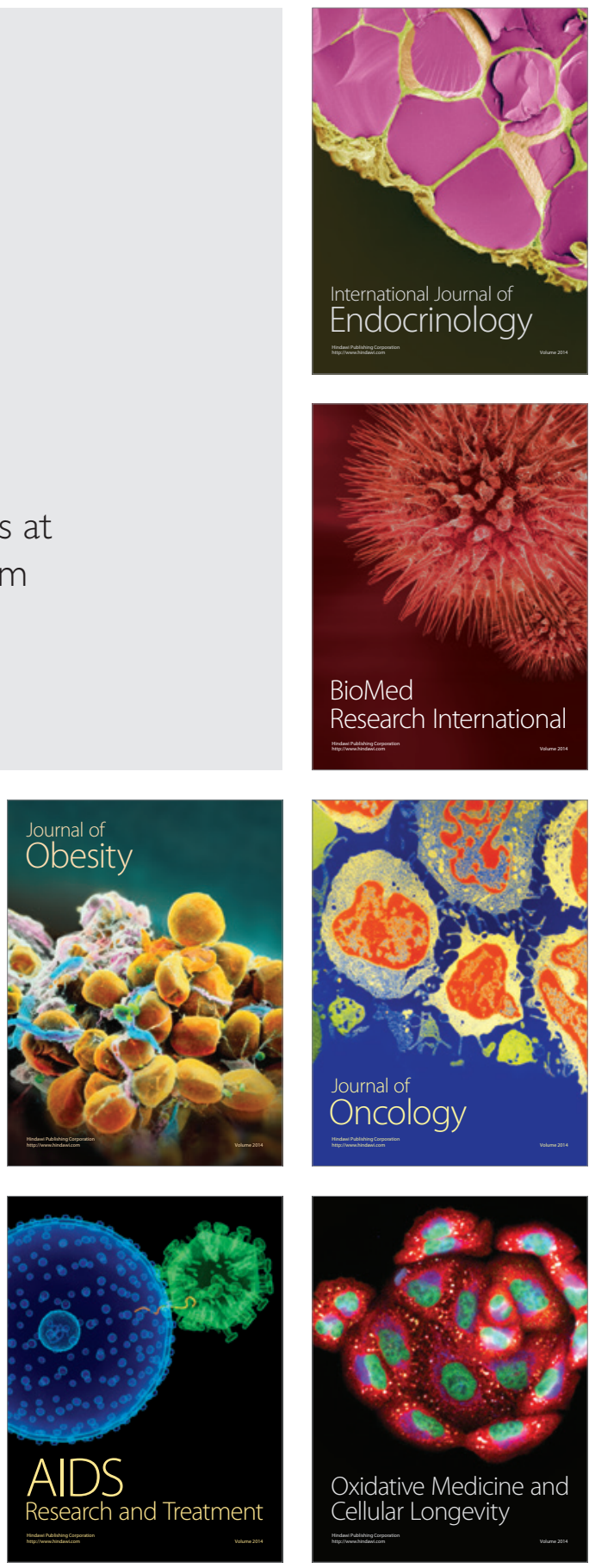